\title{
Natural variability of lung function in young healthy school children
}

\author{
Jane Kirkby ${ }^{1,2}$, Vassiliki Bountziouka ${ }^{1}$, Sooky Lum ${ }^{1}$, Angie Wade $^{3}$ and \\ Janet Stocks ${ }^{1}$ \\ Affiliations: \\ ${ }^{1}$ Respiratory, Critical Care and Anaesthesia section in Portex Unit, University College London, Institute of \\ Child Health, London, UK. \\ ${ }^{2}$ Great Ormond Street Hospital for Children NHS Foundation Trust, London, UK. \\ ${ }^{3}$ Population, Policy and Practice Programme, University College London, Institute of Child Health, London, UK.
}

\section{Correspondence:}

Jane Kirkby, Respiratory, Critical Care and Anaesthesia section, Portex Unit, UCL Institute of Child Health, 30 Guilford Street, London, WC1N 1EH, UK.

E-mail: J.kirkby@ucl.ac.uk

ABSTRACT Knowledge about long-term variability of lung function in healthy children is essential when monitoring and treating those with respiratory disease over time. The aim of this study was to define the natural variability in spirometry in young children after an interval of 12 months.

The Size and Lung function In Children study was a prospective study designed to assess spirometry and body size, shape and composition in a multi-ethnic population of London school children. 14 schools with a wide range of socioeconomic circumstances were recruited. Spirometric and anthropometric assessments and parental questionnaires pertaining to respiratory symptoms, previous medical history, pubertal status and socioeconomic circumstances were completed at baseline and $\sim 1$ year later.

Technically acceptable spirometry data on two occasions $\sim 1$ year apart (range 9-16 months) were available in 758 children (39\% boys, mean \pm SD age $8.1 \pm 1.6$ years), 593 of whom were classified as "healthy". Mean \pm SD within-subject between-test variability was $0.05 \pm 0.6 \mathrm{z}$-scores, with $95 \%$ of all the children achieving a between-test variability within $\pm 1.2 \mathrm{z}$-scores (equating to $\sim 13 \%$ predicted).

Natural variations of up to $1.2 \mathrm{z}$-scores occur in healthy children over $\sim 1$ year. These must be considered when interpreting results from annual reviews in those with lung disease who are otherwise stable, if unnecessary further investigations or changes in treatment are to be avoided.

@ERSpublications

Spirometry can vary by up to $1.2 \mathrm{z}$-scores over the course of 1 year in children without any overt lung disease http://ow.ly/YZ7mT

Received: Oct 302015 | Accepted after revision: Feb 252016 | First published online: April 132016

Support statement: This work was supported by the Wellcome Trust (WT094129MA), Asthma UK (10/013) and the Child Growth Foundation. The Size and Lung function In Children (SLIC) study team acknowledges the support of the National Institute for Health Research (NIHR), through the Comprehensive Clinical Research Network and the NIHR Biomedical Research Centre at Great Ormond Street Hospital for Children NHS Foundation Trust and University College London. Funding information for this article has been deposited with FundRef.

Conflict of interest: None declared.

Copyright @ERS 2016 


\section{Introduction}

Spirometry assessments are important both for diagnosing lung disease and for monitoring disease progression and/or response to treatment [1-4]. While comparison of an individual spirometry result with an appropriate reference or predicted value may identify abnormal lung function, it is often more clinically valuable to assess changes in an individual's lung function following an intervention or over time [5]. Differentiating true clinical changes in pulmonary status from intrinsic between-test variability can, however, be challenging.

Within-test variability and daily repeatability has been reported to be $\sim 5 \%$ in adults [6] and up to $10 \%$ in young children [3], although some studies with careful quality control and trained subjects have reported within-test variability values as low as $2 \%$ in school-aged children [7]. While such variability can be controlled to a great extent by using standardised, well calibrated equipment and adhering to published guidelines on data collection, analysis and quality control $[3,4]$, identification of clinically important changes over time requires knowledge of normal changes in lung function in health, due to growth, development or ageing. The recently published Global Lung Function Initiative (GLI) spirometry reference equations adjust for the main determinants of lung function (age, height, sex and ethnicity) and for age-dependent variability [8], as well as incorporating the important changes in the forced expiratory volume in $1 \mathrm{~s}$ (FEV1)/forced vital capacity (FVC) ratio that occur during adolescence [9]. These equations, were, however, based on cross-sectional data from 74187 individuals of different ages, and do not necessarily reflect individual changes over time [8]. Although GLI equations have been shown to be applicable in cross-sectional studies [10-13], their applicability in longitudinal studies has not been investigated.

In contrast to adults, in whom there is a steady age-related decline in lung function [5], which is often accelerated by disease [14], absolute lung function increases during childhood, with substantial changes in the pattern of lung and airway growth during puberty, such that lung disease may present as a reduced rate of lung growth, after allowing for the effects of age and somatic growth, rather than an absolute decline in overall function. Identification of clinically relevant changes over time in children with lung disease is therefore dependent not only on availability of suitable reference equations but on knowing the expected long-term variability of lung function in healthy children of similar age. While many studies have reported longitudinal changes in lung function either in relation to specific diseases [15-17] or when investigating potential impact of early life insults [18-22], there is a relative dearth of information regarding natural variability of lung function over time, especially in young children.

The aim of this study was to define long-term variability in spirometric lung function in a multi-ethnic population of London primary school children (aged 5-11 years) after an interval of $\sim 12$ months, and to investigate whether factors such as age, puberty, prior history of asthma or mild current respiratory symptoms affected such variability. We hypothesised that, while there should be no mean change in spirometry outcomes over this interval when expressed as z-scores using the GLI multi-ethnic reference equations, the long-term variability over 1 year in young children could be at least 10\%, as previously reported in adults [5].

\section{Methods}

The Size and Lung function In Children (SLIC) study was a prospective study designed to assess spirometry and body size, shape and composition in a population of multi-ethnic children (aged 5-11 years) in London schools [12]. The study protocol, methods of recruitment and details of exclusion/inclusion criteria have been published previously [12]. In brief, 14 schools with a wide range of socioeconomic circumstances were recruited. While spirometry and anthropometry were assessed in all children with written parental consent, data from those with current/chronic lung disease, e.g. sickle cell disease, cystic fibrosis and current asthma, or with congenital abnormalities likely to have an impact on lung development, were excluded from subsequent analyses $[12,13]$. All children were well enough to attend school, but were documented as "symptomatic" if a mild cough or runny nose was observed during the measurements. When possible, both spirometry and anthropometry were repeated $\sim 12$ months after the initial assessments.

The same team of paediatric respiratory physiologists carried out all spirometric assessments on both test occasions using identical equipment (Easy-on-PC; ndd Medical, Zurich, Switzerland) and standardised protocols in accordance with published guidelines [4], with subsequent over-read by a senior respiratory physiologist to ensure appropriate quality control [23]. To facilitate high quality data collection, all operators were trained and assessed to ensure that they would enthusiastically encourage each child to achieve maximal inhalation and exhalation. Furthermore, plenty of time was allowed for a full explanation, practical demonstration and subsequent practice with the child prior to commencing the tests.

Results were expressed as z-scores using the GLI spirometry reference equations, which adjust for sex, age, height and ethnicity [8]. Age was recorded in decimal years [24]. Height and weight were measured without shoes to the nearest $0.1 \mathrm{~cm}$ and $0.1 \mathrm{~kg}$, respectively (Harpenden stadiometer (Holtain Ltd, Crosswell, UK) 
and Tanita BWB600 (Tanita Corporation, Tokyo, Japan)), and, together with body mass index (BMI), were converted to z-scores to adjust for sex and age [25].

A questionnaire pertaining to respiratory symptoms, previous medical history, pubertal status (if aged $>8$ years) and socioeconomic circumstances (defined using the English Index of Multiple Deprivation (IMD) score [26] based on home postcode) was administered on both test occasions [27]. Methods of classifying puberty have been described previously [28]. In brief, children were classified as "non-pubertal" if their parents responded negatively to the relevant questions on both test occasions, or as "approaching puberty" if parents responded positively either on both occasions or just the second. Socioeconomic circumstances based on IMD 2010 score were grouped into three categories reflecting high (least deprived areas; first/second quintile), moderate (third/fourth quintile) and low socioeconomic circumstances (most deprived areas; fifth quintile) [26]. Children were broadly categorised as white (European ancestry), black (African or Caribbean descent), South Asian (originating from the Indian subcontinent) or of "other" or "mixed" ethnicities, based on parental report.

The study was guided by a steering committee and approved by the London-Hampstead research ethics committee (approval number 10/H0720/53). Parental written consent and child verbal assent were obtained prior to assessment.

\section{Data management and statistical analysis}

Results are presented as mean \pm SD for continuous variables or frequencies (\%) for categorical variables. To adjust for differences in growth and development (age, sex and height), lung function results were expressed as z-scores. Mean differences in lung function z-scores between test occasions were calculated by subtracting values of the second test from those of the first (i.e. T1-T2), with the $95 \%$ limits of these differences calculated as mean $_{\text {difference }} \pm 1.96 \mathrm{SD}_{\text {difference. }}$. This is termed the "between-test variability". When using the GLI equations in children aged 5-11 years, a difference of $1 \mathrm{z}$-score in FEV1 or FVC equates to $\sim 11 \%$ predicted [8]. For the purposes of this study, and to aid clinical interpretation, between-test variability was classified as low for those with differences of $\leqslant 0.5 \mathrm{z}$-scores, medium if $>0.5$ and $\leqslant 1 \mathrm{z}$-score, high if $>1$ and $\leqslant 2 \mathrm{z}$-scores and extreme if $>2 \mathrm{z}$-scores. The Pearson correlation coefficient (with $95 \% \mathrm{CI}$ ) was used to assess the magnitude of any association between changes in lung function and height $\mathrm{z}$-scores between test occasions. Both linear and logistic regression models were used to evaluate the association of growth factors (i.e. age, height, "approaching puberty") and difference in lung function z-scores between tests. Logistic regression models with 95\% CI described any increased odds of having a difference in lung function between test occasions that exceeded $\pm 0.5 \mathrm{z}$-scores, while the linear regression models evaluated the association of growth factors (e.g. time interval between tests, onset of puberty, change in height $\mathrm{z}$-score) with the change in lung function z-scores. Since results were similar using both methods, logistic regression models were presented to aid clinical interpretation.

Analyses were performed using SPSS v.20 (IBM Corp., Armonk, NY, USA).

\section{Results}

Of the 1901 subjects assessed in the main study [12], measurements were only possible on a single test occasion in 1120, due to lack of facilities in some schools for repeat testing within a specified time period, children moving to senior schools or lack of parental consent on one or other test occasion. Results from $23(2.9 \%)$ out of 781 children with repeated measurements were excluded due to poor quality spirometry or change in asthma health status between test occasions.

Technically acceptable data on two test occasions 9-16 months apart (mean 11.0 months) were available in 758 children, hereafter referred to as "all subjects", a group which comprised 39\% boys, with mean \pm SD age on first test occasion of $8.1 \pm 1.6$ years. Of these, $63(8 \%)$ had been born preterm and/or with low birthweight, 37 (5\%) had been previously diagnosed with asthma and $65(8.6 \%)$ had mild respiratory symptoms on one or other test occasion (4\% on first test and $4.6 \%$ on second test). $44 \%$ of "all subjects" were white, $23 \%$ black, $21 \%$ South Asian and 12\% of "other/mixed" ethnicities. Apart from an increased proportion of preterm/low birthweight and a reduced proportion of asthma among South Asians, a similar ethnic distribution was observed across all subgroups. With the exception of a small reduction in the proportion of boys, background characteristics of children undergoing repeated measurements were very similar to those observed for the entire SLIC population of 1901 children [12, 27]. Background characteristics of "all subjects" as well as the various subgroups were also very similar to those of the "healthy population", which comprised the 593 (78\%) children with repeat measurements who had no significant prior or current medical history (figure 1 and table 1 ).

Between $14 \%$ and $24 \%$ of children in the various populations/subgroups were "approaching puberty" by the second test occasion. Socioeconomic circumstances as classified by the IMD 2010 score were consistent 


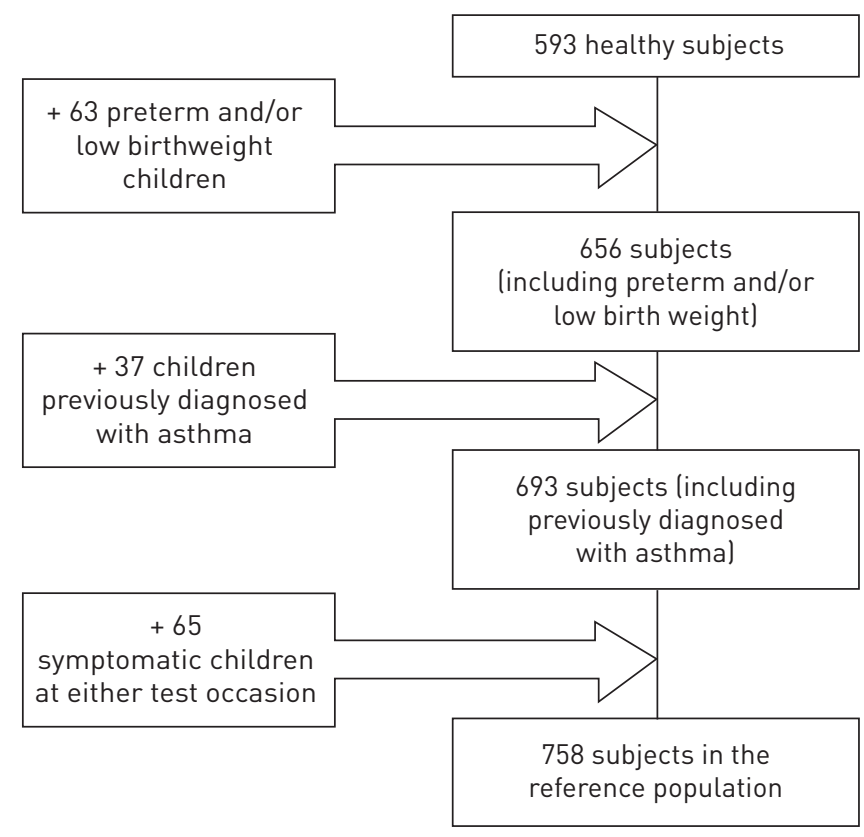

FIGURE 1 Flow chart for subjects included in the study.

between test occasions in all but $4 \%$ of children, the latter being classified for the purposes of this study using information recorded on the second test occasion. One third of both "all subjects" and "healthy children", and nearly half of those born preterm or with "previous asthma" were classified within the lower (fifth) quintile according to IMD 2010. There were no significant differences in either anthropometry or lung function between "all subjects" and the healthy population, although those with "prior asthma" tended to have a higher BMI than other groups and, together with those who were symptomatic on the day of test, tended to have slightly lower lung function (table 1).

After an interval of 9-16 months, the mean \pm SD between-test variability of FEV1 was $0.05 \pm 0.6 \mathrm{z}$-scores for "all subjects". Two-thirds of "all subjects" as well as "healthy" children had low variability ( $\pm 0.5 \mathrm{z}$-scores, i.e. $<6 \%$ predicted), with similar results for children born preterm/low birthweight or with prior asthma, although this proportion was slightly lower (54\%) in those who were symptomatic on one or other test occasion (table 2$)$. Between-test variability of $\pm 1.2 \mathrm{z}$-scores $(\sim 13 \%$ predicted) was achieved by $95 \%$ of all young children included in the study, with only four children $(0.5 \%)$ having extreme variability between test occasions ( $>2 \mathrm{z}$-scores) (table 2 ). Although results from children with the "extreme variability" were re-analysed to verify that this was not due to any issues with quality control, this did not lead to any further exclusion. Those with lung function below the fifth centile on the initial visit (less than

TABLE 1 Participants' characteristics

\begin{tabular}{|c|c|c|c|c|c|c|c|c|c|c|}
\hline & \multicolumn{2}{|c|}{ Healthy } & \multicolumn{2}{|c|}{ Preterm/LBW } & \multicolumn{2}{|c|}{ Prior asthma } & \multicolumn{2}{|c|}{ Symptomatic } & \multicolumn{2}{|c|}{ All subjects ${ }^{\#}$} \\
\hline & Test 1 & Test 2 & Test 1 & Test 2 & Test 1 & Test 2 & Test 1 & Test 2 & Test 1 & Test 2 \\
\hline Subjects $\mathrm{n}$ & \multicolumn{2}{|c|}{593} & \multicolumn{2}{|c|}{63} & \multicolumn{2}{|c|}{37} & \multicolumn{2}{|c|}{65} & \multicolumn{2}{|c|}{758} \\
\hline Male \% & \multicolumn{2}{|c|}{39} & \multicolumn{2}{|c|}{43} & \multicolumn{2}{|c|}{38} & \multicolumn{2}{|c|}{40} & \multicolumn{2}{|c|}{39} \\
\hline Age years & $8.1 \pm 1.6$ & $9.1 \pm 1.6$ & $8.6 \pm 1.4$ & $9.5 \pm 1.4$ & $8.1 \pm 1.6$ & $9.1 \pm 1.6$ & $7.7 \pm 1.4$ & $8.7 \pm 1.5$ & $8.1 \pm 1.6$ & $9.1 \pm 1.6$ \\
\hline z-height ${ }^{7}$ & $0.40 \pm 1.0$ & $0.48 \pm 1.0$ & $0.14 \pm 1.2$ & $0.24 \pm 1.5$ & $0.63 \pm 1.1$ & $0.67 \pm 1.1$ & $0.29 \pm 1.1$ & $0.37 \pm 1.1$ & $0.38 \pm 1.0$ & $0.46 \pm 1.0$ \\
\hline z-weight ${ }^{\pi}$ & $0.44 \pm 1.1$ & $0.50 \pm 1.1$ & $0.25 \pm 1.5$ & $0.40 \pm 1.5$ & $0.80 \pm 1.1$ & $0.82 \pm 1.2$ & $0.42 \pm 1.3$ & $0.46 \pm 1.3$ & $0.44 \pm 1.2$ & $0.50 \pm 1.2$ \\
\hline z-BMI & $0.31 \pm 1.2$ & $0.35 \pm 1.2$ & $0.24 \pm 1.5$ & $0.38 \pm 1.6$ & $0.63 \pm 1.4$ & $0.63 \pm 1.4$ & $0.36 \pm 1.4$ & $0.38 \pm 1.4$ & $0.32 \pm 1.3$ & $0.37 \pm 1.3$ \\
\hline FEV 1 L & $1.5(0.5-2.8)$ & $1.7(0.8-3.8)$ & $1.5(0.8-2.5)$ & $1.7(0.8-2.9)$ & $1.5(0.8-2.1)$ & $1.6(0.9-2.4)$ & $1.5(1.1-2.5)$ & 1.8 (1.2-2.7) & $1.5[0.5-2.8]$ & $1.7(0.8-3.8)$ \\
\hline FVC L & $1.8(0.6-3.4)$ & $2.0(0.8-4.6)$ & $1.8(0.9-2.9)$ & $2.0(1.1-3.2)$ & $1.7(0.9-2.4)$ & $2.0(1.1-2.8)$ & $1.8(1.2-2.6)$ & $2.1(1.4-3.1)$ & $1.8[0.6-3.4]$ & $2.0(0.8-4.6)$ \\
\hline $\mathrm{z}-\mathrm{FEV}_{1}{ }^{+}$ & $0.05 \pm 0.92$ & $-0.02 \pm 0.92$ & $-0.03 \pm 0.85$ & $-0.04 \pm 0.91$ & $-0.35 \pm 0.8$ & $-0.40 \pm 0.9$ & $-0.23 \pm 1.0$ & $-0.20 \pm 1.0$ & $0.00 \pm 0.94$ & $-0.05 \pm 0.92$ \\
\hline z-FVC ${ }^{+}$ & $0.19 \pm 0.92$ & $0.15 \pm 0.89$ & $0.18 \pm 0.99$ & $0.13 \pm 0.92$ & $0.00 \pm 0.8$ & $0.00 \pm 0.6$ & $0.10 \pm 1.0$ & $0.18 \pm 1.0$ & $0.17 \pm 0.92$ & $0.14 \pm 0.89$ \\
\hline z-FEV $1 / F^{\prime} C^{+}$ & $-0.27 \pm 0.97$ & $-0.34 \pm 0.87$ & $-0.39 \pm 0.91$ & $-0.31 \pm 0.85$ & $-0.70 \pm 0.8$ & $-0.78 \pm 0.9$ & $-0.56 \pm 1.2$ & $-0.63 \pm 1.0$ & $-0.32 \pm 0.98$ & $-0.38 \pm 0.89$ \\
\hline
\end{tabular}

Data are presented as mean \pm SD or mean (range), unless otherwise stated. LBW: low birthweight; BMI: body mass index; FEV1: forced expiratory volume in $1 \mathrm{~s}$; FVC: forced vital capacity. Note that when interpreting paediatric spirometry, results need to be adjusted for age, sex, height and ethnicity using z-scores, since in the presence of ongoing growth, changes cannot be based on absolute values. \#: includes all children (i.e. healthy+preterm/LBW+prior asthma+symptomatic at test); "1: calculated using British 1990 growth reference centiles [25]; ${ }^{+}$: calculated using all-age multi-ethnic reference equations (Global Lung Function Initiative 2012) [8]. 


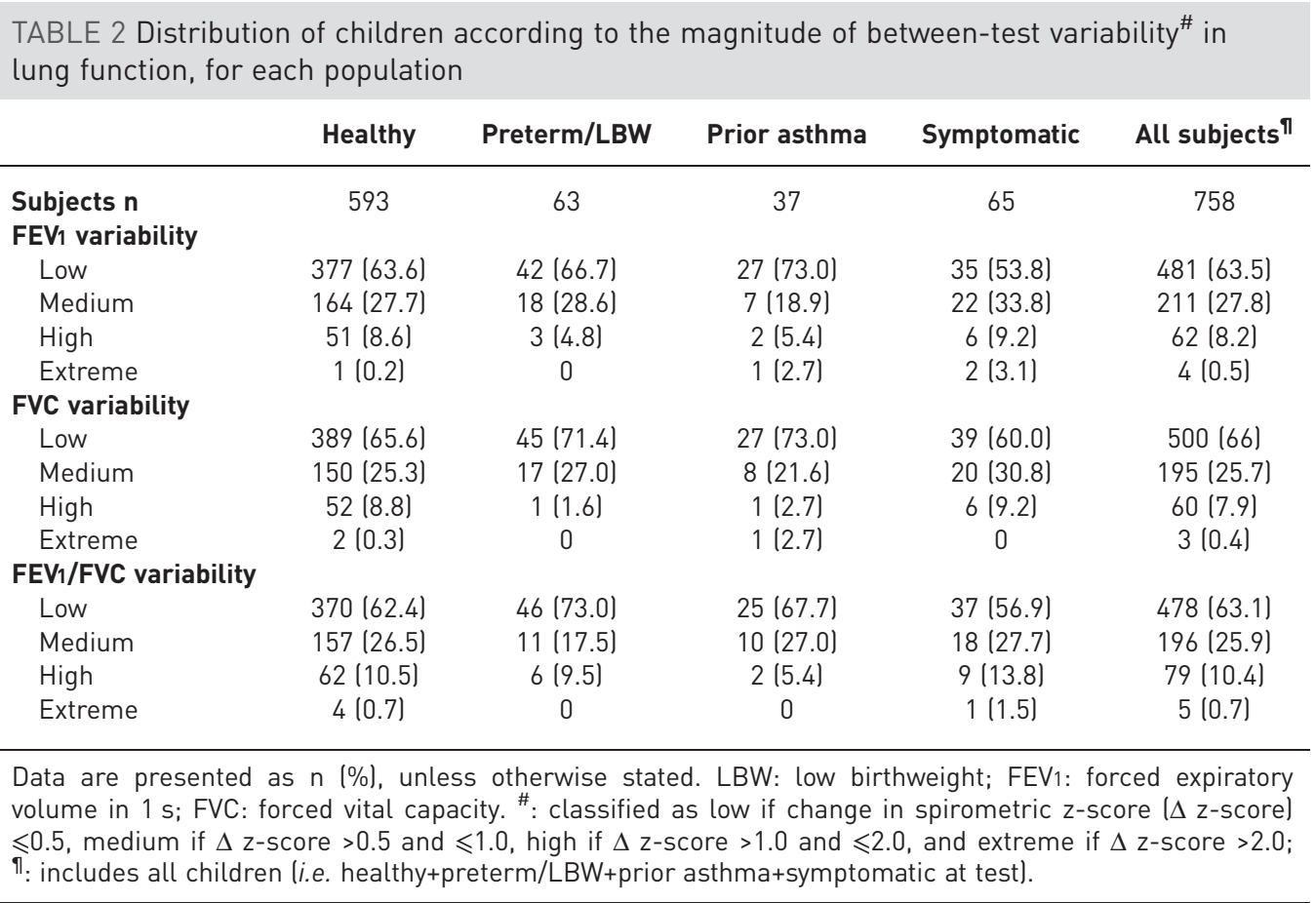

$-1.64 \mathrm{z}$-scores) tended to have larger increases in lung function when re-tested than those in whom results were within the normal range (figure 2 ); however, there was only a weak correlation $\left(\mathrm{r}^{2}=0.09\right)$ between the magnitude of change over time and lung function on the first occasion.

There was no association between changes in z-FEV1 and changes in height z-score between test occasions among "all subjects" (rho=0.09, 95\% CI 0.02-0.16), suggesting that the more extreme changes in $\mathrm{z}-\mathrm{FEV} 1$ seen in a few children were not associated with the sudden growth spurts that may occur at the start of puberty (figure 3 ). Similar results were found across all ethnic groups and for FVC and FEV1/FVC (data not shown).

Inclusion of children born preterm/low birthweight, previously diagnosed with asthma or symptomatic at test occasion within the "all subjects" population had minimal effect on the distribution of $\mathrm{z}$-score variability over time; mean \pm SD differences in FEV1 $\mathrm{z}$-scores were $0.07 \pm 0.59$ in the healthy population and $0.05 \pm 0.60$ for "all subjects", with similar values for FVC and FEV1/FVC (table 3 ).

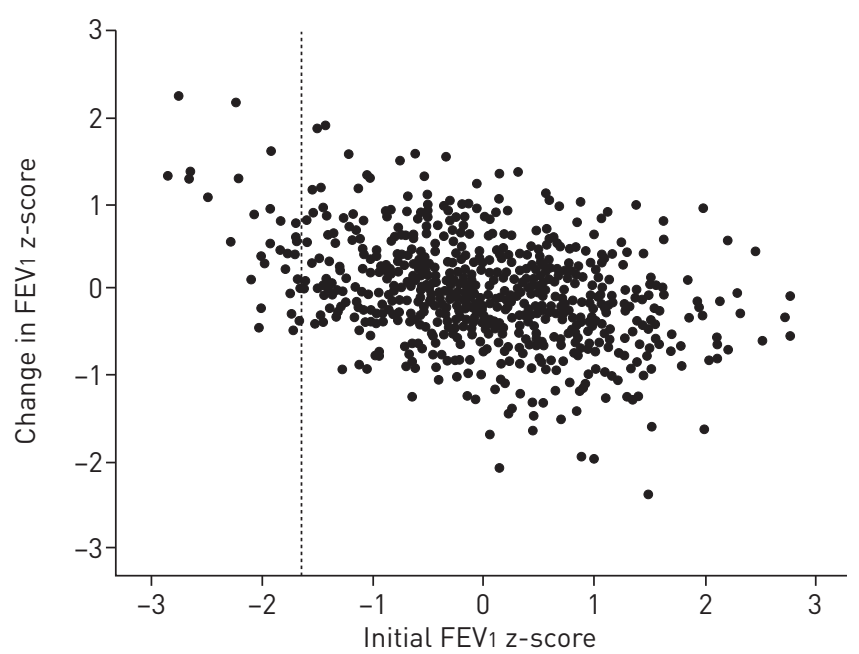

FIGURE 2 Change in forced expiratory volume in $1 \mathrm{~s}$ (FEV 1 ) z-score (visit 2-visit 1) compared with the FEV1 $z$-score on the initial visit. The dashed line indicates the lower limit of normal (-1.64 z-scores, fifth centile). 


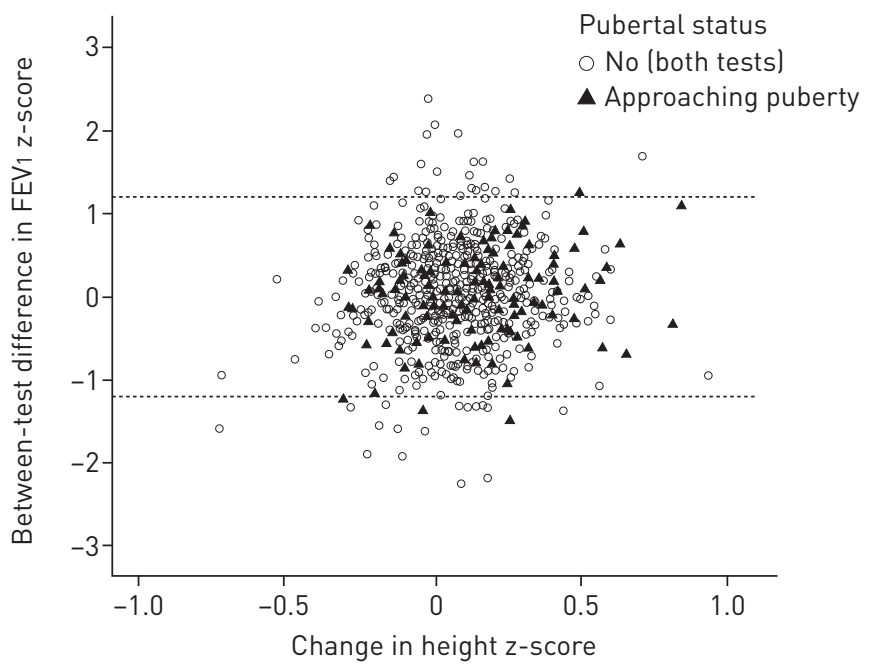

FIGURE 3 Between-test (T1-T2) difference in forced expiratory volume in $1 \mathrm{~s}(\mathrm{FEV} 1) \mathrm{z}$-scores versus change in height z-score (T2-T1), according to pubertal status for the reference population. Dashed lines indicate the $95 \%$ limits of agreement ( $\pm 1.2 \mathrm{z}$-scores). The lack of association between differences in FEV 1 z-scores and height z-scores, and negligible effects of pubertal status, suggest that these factors have a minimal role in explaining the more extreme changes seen in a few children.

Although children "approaching puberty" had a slightly higher odds of z-FEV1 changing by $>0.5 \mathrm{z}$-scores over the year, this was not statistically significant (odds ratio $1.21,95 \%$ CI $0.76-1.93$ ), with similar results for FVC and FEV1/FVC (table 4). After adjustment for age, time interval, pubertal status, sex and ethnicity, change in height z-score was only associated with an increased odds of z-FVC changing by $>0.5 \mathrm{z}$-scores per unit change in $z$-height (table 4). Results were similar when analysed as a continuum (data not shown). The magnitude of variability in lung function was independent of socioeconomic circumstances, as measured using IMD 2010 (data not shown) after adjusting for age, sex, height and pubertal status.

\section{Discussion}

In this study we performed repeated assessments of spirometry and anthropometry after an interval of 9-16 months in a large, multi-ethnic population of London primary school children. Although the follow-up for this study was limited to $\sim 1$ year for practical reasons, this represented a clinically meaningful interval, since children with lung disease frequently undergo detailed annual assessment (the "annual review") in addition to more regular assessments throughout the year [29]. Use of the GLI ethnic-specific spirometry reference equations [8] was appropriate for interpreting lung function in this population, with mean \pm SD results approximating $0 \pm 1 \mathrm{z}$-scores on both test occasions. While two-thirds of the children demonstrated relatively low variability in spirometric $\mathrm{z}$-scores (i.e. values differed by $<0.5 \mathrm{z}$-scores ( $6 \%$ predicted) when assessed a year apart), changes of up to $1.2 \mathrm{z}$-scores (13\% predicted) were encountered among $95 \%$ of the population. Although neither unusual changes in height nor transition into puberty were predictive of increased variability, there was a very weak inverse correlation between magnitude of change over time and initial lung function, this relationship being influenced primarily by the few children with lung function below the fifth centile on the first occasion. This could reflect either slight underperformance by a few children on the first occasion or

TABLE 3 Mean differences between the two test occasions in lung function z-scores for all populations tested

\begin{tabular}{lcrrr} 
& Subjects $\mathbf{c}$ & z-FEV & z-FVC & z-FEV $1 / F V C$ \\
\hline Healthy & 593 & $0.07 \pm 0.59$ & $0.04 \pm 0.58$ & $0.07 \pm 0.66$ \\
Preterm/LBW & 63 & $0.01 \pm 0.53$ & $0.05 \pm 0.48$ & $-0.08 \pm 0.60$ \\
Prior asthma & 37 & $0.05 \pm 0.62$ & $0.00 \pm 0.57$ & $0.08 \pm 0.49$ \\
Symptomatic & 65 & $-0.04 \pm 0.74$ & $-0.08 \pm 0.63$ & $0.07 \pm 0.75$ \\
All subjects $^{\#}$ & 758 & $0.05 \pm 0.60$ & $0.03 \pm 0.58$ & $0.06 \pm 0.66$ \\
\hline
\end{tabular}

Data are presented as mean \pm SD differences (T1-T2), unless otherwise stated. $F E V_{1}$ : forced expiratory volume in $1 \mathrm{~s}$; FVC: forced vital capacity; LBW: low birthweight. \#: includes all children (i.e. healthy+ preterm/LBW+prior asthma+symptomatic at test). 
TABLE 4 Growth factors associated with the adjusted odds of having difference in lung function (T1-T2) >0.5 z-scores between test occasions

\begin{tabular}{llcc} 
& $\Delta \mathbf{z}-\mathrm{FEV} \mathbf{1}>\mathbf{0 . 5}$ & $\Delta \mathbf{z}-\mathrm{FVC}>\mathbf{0 . 5}$ & $\Delta \mathbf{z}-\mathrm{FEV} \mathbf{5} / \mathrm{FVC}>\mathbf{0 . 5}$ \\
\hline Age (at T1), per year & $0.98(0.88-1.09)$ & $0.86(0.77-0.96)$ & $0.81(0.73-0.91)$ \\
Time interval between measurements, per month & $1.01(0.95-1.09)$ & $1.01(0.94-1.09)$ & $1.08(1.01-1.16)$ \\
Difference in height z-score (T2-T1), per unit & $1.03(0.48-2.22)$ & $\mathbf{2 . 5 1 ( 1 . 1 4 - 5 . 5 4 )}$ & $0.60(0.28-1.31)$ \\
Approaching puberty versus no puberty & $1.21(0.76-1.93)$ & $1.04(0.64-1.71)$ & $1.18(0.73-1.92)$
\end{tabular}

Data are presented as odds ratios $(95 \% \mathrm{CI}) . \Delta \mathrm{z}$ : change in $\mathrm{z}$-scores; FEV1: forced expiratory volume in 1 s; FVC: forced vital capacity. Models included all variables in the table and were additionally adjusted for sex and ethnicity. Bold indicates statistical significance.

undetected respiratory symptoms. However, the inclusion of children with mild current symptoms, prior wheeze or low birthweight did not influence either mean spirometry results [13] or the magnitude of change over time. To avoid potential over-treatment or unnecessary additional investigations in otherwise stable children, the natural year-to-year variability of lung function that can occur in the absence of any overt lung disease needs to be considered when interpreting results from annual reviews in children with lung disease.

Baseline and longitudinal spirometric assessments are an integral part of routine care and clinical decision-making for many respiratory diseases [1-4]. For example, in cystic fibrosis, monitoring disease progression can predict mortality and indicate need for intervention [29]. Liou et al. [29] recently reported relatively large individual annual decreases in FEV1 \% predicted, with $>20 \%$ of patients having decreases $\geqslant 5 \%$ predicted. They highlight the importance of considering individual annual changes as well as aggregated population changes, appropriate interpretation of which requires knowledge of normal year-to-year variability.

In this study, after an interval of $\sim 1$ year, between-test variability within $\pm 1.2 \mathrm{z}$-scores (approximately $\pm 13 \%$ predicted) was achieved by $95 \%$ of young children, with only five children (0.7\%) having extreme variability between test occasions ( $>2 \mathrm{z}$-scores). This is in keeping with proposals by the American Thoracic Society and European Respiratory Society, which suggested that when interpreting within-subject annual changes, those $>15 \%$ were indicative of a clinically meaningful change in adults [5]. This study of young school-aged children demonstrates that, provided close attention is paid to quality control, even in large field studies that include some mildly symptomatic subjects, not only are between-test variability scores of FEV1 and FVC similar to each other, but overall variation is similar to that reported in adults. It must, however, be noted that the degree of variability observed in this study, where all assessments were undertaken by specialist staff using a highly standardised protocol, is likely to be considerably less than may occur in primary care, where lung function may be measured under less favourable conditions. Although the magnitude of change by the second test was slightly elevated in the few children whose initial results fell below the normal range, the majority of children in this study had results within the normal range, and among these children there was no relationship between magnitude of change and baseline lung function.

Expressing differences in lung function over time as z-scores as in this study, rather than absolute changes $[20,21]$, ensured appropriate adjustments were made for growth [8]. Neither change in height z-score nor pubertal status was associated with increased between-test variability of lung function, suggesting that the GLI equations adequately take such factors into account in young children. The current findings are, however, limited to children aged $<12$ years and, since only a relatively small proportion of these children were "approaching puberty", we were not able to explore the differential effects of puberty on patterns of lung and airway growth, which remain poorly understood. Although the assumption that longitudinal changes in this study follow predicted changes from the cross-sectional GLI data must be applied cautiously, the use of the GLI reference equations appeared to have been appropriate in this longitudinal study, and provided a reliable index that remained constant during growth. The stability of group mean spirometric z-scores over the year in this study is consistent with previous reports [30,31], although these studies did not report within-subject variability.

A further strength of the study was that all assessments were undertaken by the same specialised team of paediatric respiratory physiologists using identical equipment and standardised protocols, with strict quality control criteria on both test occasions. The use of modern equipment and strict quality control criteria will have facilitated achievement of maximum FVC in these young children. This may have contributed to the slightly higher FVC, and hence a slightly lower FEV1/FVC than predicted by the GLI equations, as well as the similar variability over time of FVC when compared with FEV1. While "learning effects" in repeated assessments cannot be excluded [5], the fact that the group mean lung function remained so stable at follow-up suggests that appropriate training and supervision were provided on both test occasions. 


\section{Conclusion}

Knowledge of the natural between-test variability of lung function in childhood is essential for appropriate interpretation of longitudinal assessments in both epidemiological studies and when monitoring individual children with lung disease. This study demonstrates that, even under optimal conditions where all assessments are undertaken by highly trained paediatric respiratory physiologists, lung function can vary by up to $1.2 \mathrm{z}$-scores over the course of 1 year in children without any overt lung disease. Such variability is likely to be even higher in many hospital outpatient clinics or primary care, and must be taken into account when interpreting results from annual reviews in those with lung disease. Appreciation of the normal variability that can occur in health may help reduce anxiety for the child or their family, who are often alarmed at even the smallest reductions in lung function during annual review. Furthermore, although clinical management should always be based on treating the patient, rather than the numerical results of any test, awareness of the intrinsic variability of lung function during annual reviews may help prevent unnecessary further investigations or changes in treatment plans for children with lung disease who are otherwise stable.

\section{Acknowledgements}

We thank Rachel Bonner, Emma Raywood, Sarah Legg, Dave Sears, Simon Lee and Philippa Cottam (all Respiratory, Critical Care and Anaesthesia section, University College London, Institute of Child Health, London, UK) for their help with data collection and data management. We would particularly like to thank the head teachers and staff of participating schools for facilitating the recruitment and school assessments, and last but not least the children and families who participated in the study.

\section{References}

1 Banasiak NC. Spirometry in primary care for children with asthma. Pediatr Nurs 2014; 40: 195-198.

2 Stocks J, Kirkby J, Lum S. How to avoid misinterpreting lung function tests in children: a few practical tips. Paediatr Respir Rev 2014; 15: 170-180.

3 Beydon N, Davis SD, Lombardi E, et al. An official American Thoracic Society/European Respiratory Society statement: pulmonary function testing in preschool children. Am J Respir Crit Care Med 2007; 175: 1304-1345.

Miller MR, Hankinson J, Brusasco V, et al. Standardisation of spirometry. Eur Respir J 2005; 26: 319-338.

5 Pellegrino R, Viegi G, Brusasco V, et al. Interpretative strategies for lung function tests. Eur Respir J 2005; 26: 948-968.

6 Enright PL, Beck KC, Sherrill DL. Repeatability of spirometry in 18,000 adult patients. Am J Respir Crit Care Med 2004; 169: 235-238.

7 Pérez-Padilla R, Regalado-Pineda J, Mendoza L, et al. Spirometric variability in a longitudinal study of school-age children. Chest 2003; 123: 1090-1095.

8 Quanjer PH, Stanojevic S, Cole TJ, et al. Multi-ethnic reference values for spirometry for the 3-95-yr age range: the global lung function 2012 equations. Eur Respir J 2012; 40: 1324-1343.

9 Quanjer PH, Borsboom GJ, Kivastik J, et al. Cross-sectional and longitudinal spirometry in children and adolescents: interpretative strategies. Am J Respir Crit Care Med 2008; 178: 1262-1270.

10 Bonner R, Lum S, Stocks J, et al. Applicability of the global lung function spirometry equations in contemporary multiethnic children. Am J Respir Crit Care Med 2013; 188: 515-516.

11 Hall GL, Thompson BR, Stanojevic S, et al. The Global Lung Initiative 2012 reference values reflect contemporary Australasian spirometry. Respirology 2012; 17: 1150-1151.

12 Lum S, Bountziouka V, Sonnappa S, et al. Lung function in children in relation to ethnicity, physique and socioeconomic factors. Eur Respir J 2015; 46: 1662-1671.

13 Lum S, Bountziouka V, Sonnappa S, et al. How "healthy" should children be when selecting reference samples for spirometry? Eur Respir J 2015; 45: 1576-1581.

14 Tai A, Tran H, Roberts $\mathrm{M}$, et al. The association between childhood asthma and adult chronic obstructive pulmonary disease. Thorax 2014; 69: 805-810.

15 Welsh L, Robertson CF, Ranganathan SC. Increased rate of lung function decline in Australian adolescents with cystic fibrosis. Pediatr Pulmonol 2014; 49: 873-877.

16 Kurukulaaratchy RJ, Raza A, Scott M, et al. Characterisation of asthma that develops during adolescence; findings from the Isle of Wight Birth Cohort. Respir Med 2012; 106: 329-337.

17 Lum S, Kirkby J, Welsh L, et al. Nature and severity of lung function abnormalities in extremely pre-term children at 11 years of age. Eur Respir J 2011; 37: 1199-1207.

18 Chan JY, Stern DA, Guerra S, et al. Pneumonia in childhood and impaired lung function in adults: a longitudinal study. Pediatrics 2015; 135: 607-616.

19 Kotecha SJ, Watkins WJ, Henderson AJ, et al. The effect of birth weight on lung spirometry in white, school-aged children and adolescents born at term: a longitudinal population based observational cohort study. J Pediatr 2015; 166: $1163-1167$.

20 Piccioni P, Tassinari R, Carosso A, et al. Lung function changes from childhood to adolescence: a seven-year follow-up study. BMC Pulm Med 2015; 15: 31.

21 Lodge CJ, Lowe AJ, Allen KJ, et al. Childhood wheeze phenotypes show less than expected growth in FEV 1 across adolescence. Am J Respir Crit Care Med 2014; 189: 1351-1358.

22 Stern DA, Morgan WJ, Wright AL, et al. Poor airway function in early infancy and lung function by age 22 years: a non-selective longitudinal cohort study. Lancet 2007; 370: 758-764.

23 Kirkby J, Welsh L, Lum S, et al. The EPICure study: comparison of pediatric spirometry in community and laboratory settings. Pediatr Pulmonol 2008; 43: 1233-1241.

24 Quanjer PH, Hall GL, Stanojevic S, et al. Age- and height-based prediction bias in spirometry reference equations. Eur Respir J 2012; 40: 190-197. 
25 Cole TJ, Freeman JV, Preece MA. British 1990 growth reference centiles for weight, height, body mass index and head circumference fitted by maximum penalized likelihood. Stat Med 1998; 17: 407-429.

26 McLennan D, Barnes H, Noble M, et al. The English Indices of Deprivation 2010. London, Dept for Communities and Local Government, 2011. Available from: www.gov.uk/government/uploads/system/uploads/attachment_data/ file/6320/1870718.pdf

27 Lum S, Sonnappa S, Wade A, et al. Exploring ethnic differences in lung function: the Size and Lung function In Children (SLIC) study protocol and feasibility. London, UCL Institute of Child Health, 2014. Available from: http://discovery.ucl.ac.uk/1417500/

28 Lum S, Bountziouka V, Harding S, et al. Assessing pubertal status in multi-ethnic primary schoolchildren. Acta Paediatr 2015; 104: e45-e48.

29 Liou TG, Elkin EP, Pasta DJ, et al. Year-to-year changes in lung function in individuals with cystic fibrosis. J Cyst Fibros 2010; 9: 250-256.

30 Vollsæter M, Røksund OD, Eide GE, et al. Lung function after preterm birth: development from mid-childhood to adulthood. Thorax 2013; 68: 767-776.

31 Kotecha SJ, Watkins WJ, Paranjothy S, et al. Effect of late preterm birth on longitudinal lung spirometry in school age children and adolescents. Thorax 2012; 67: 54-61. 